\title{
应用于局域表面等离激元共振扫描显微探针的球形Au@Ag纳米 粒子的合成及介电敏感性检测
}

\author{
何茜唐同丹易骏刘必聚王芳芳任 斌周剑章* \\ (厦门大学化学化工学院化学系, 固体表面物理化学国家重点实验室, 福建厦门 361005)
}

\begin{abstract}
摘要: 局域表面等离激元共振(LSPR)显微探针的检测灵敏性主要取决于针尖上修饰的纳米粒子的LSPR性 质. 本文采用阴离子辅助法, 在水溶液中通过调节 $\mathrm{Au}$ 核与 $\mathrm{Ag}^{+}$的物质的量之比, 实现Au核上不同厚度的 $\mathrm{Ag}$ 壳层 包覆, 可控地一步合成均一性好、银壳层较厚 $(\geq 10 \mathrm{~nm})$ 的核壳比不同的球形Au@Ag纳米粒子. 通过扫描电镜 (SEM)、透射电镜(TEM)及扫描透射电子显微镜X射线能谱(STM-EDS)线扫描分析对不同核壳比的Au@Ag纳 米粒子进行形貌组成表征, 证实了所合成核壳结构的可控性. 将不同核壳比的Au@Ag纳米粒子置于不同折射 率溶液中进行纳米粒子介电敏感性的研究, 表明7.5 nm Au@28 nm Ag的纳米结构具有最高的品质因子. 同时 将不同核壳比的Au@Ag纳米粒子置于不同折射率的非导电性基底上进行单颗纳米粒子散射性质的研究, 结果 表明7.5 nm Au@28 nm Ag纳米粒子适合作为LSPR显微探针的高检测灵敏性纳米结构之一.
\end{abstract}

关键词: Au@Ag纳米粒子; 阴离子辅助一步法合成; 表面等离激元共振； 介电敏感性；

单粒子的暗场显微术

中图分类号: 0646

\section{Spherical Au@Ag Nanoparticles for Localized Surface Plasmon Resonance Scanning Probes: Synthesis and Dielectric Sensitivity}

\author{
$\mathrm{HE} \mathrm{Xi} \quad$ TANG Tong-Dan YI Jun LIU Bi-Ju WANG Fang-Fang \\ REN Bin ZHOU Jian-Zhang* \\ (State Key Laboratory of Physical Chemistry of Solid Surfaces, Department of Chemistry, College of Chemistry and \\ Chemical Engineering, Xiamen University, Xiamen 361005, Fujian Province, P. R. China)
}

\begin{abstract}
The detection sensitivity of localized surface plasmon resonance (LSPR) microscopic probes is mainly determined by the LSPR property of the modified metal nanoparticle at the end of the probe. In this paper, spherical Au@Ag nanoparticles (NPs) with good size uniformity and a thick Ag shell ( $\geq 10 \mathrm{~nm})$ were synthesized using the anion-assisted one-step synthesis method in aqueous solution, and the thickness of the $\mathrm{Ag}$ shell can be controlled by simply adjusting the molar ratio of $\mathrm{Au}$ to $\mathrm{Ag}$ in the solution. We characterized the morphology and composition of Au@Ag NPs with different core-shell ratios by scanning electron microscopy (SEM), transmission electron microscopy (TEM), and scanning transmission electron microscopy-energy dispersive X-ray spectroscopy (STEM-EDS) line scanning analyses, which confirmed the controllable synthesis of Au@Ag core-shell NPs by this method. Measurement of the dielectric sensitivity of Au@Ag NPs with different core-shell ratios in different refractive index solutions showed that the core-shell nanostructure of $7.5 \mathrm{~nm} \mathrm{Au@28} \mathrm{nm} \mathrm{Ag} \mathrm{has} \mathrm{the} \mathrm{highest} \mathrm{figure} \mathrm{of} \mathrm{merit} \mathrm{for} \mathrm{detection.} \mathrm{Further} \mathrm{investigation} \mathrm{of} \mathrm{the} \mathrm{plasmonic}$
\end{abstract}

Received: March 19, 2015; Revised: June 4, 2015; Published on Web: June 4, 2015.

"Corresponding author. Email: jzzhou@xmu.edu.cn; Tel: +86-592-2189663.

The project was supported by the National Natural Science Foundation of China $(21273182,21321062,512053333)$

国家自然科学基金(21273182,21321062, 512053333)资助项目

(C) Editorial office of Acta Physico-Chimica Sinica 
properties of a single Au@Ag NP on nonconductive substrates with different refractive indexes confirmed that 7.5 nm Au@28 nm Ag NPs are one of the most suitable candidates for dielectric sensing in LSPR microscopy among the spherical Au@Ag NPs.

Key Words: Au@Ag nanoparticle; Anion-assisted one-step synthesis; Surface plasmon resonance scanning microscopy; Dielectric sensitivity; Dark-filed microscopy of single nanoparticle

\section{1 引言}

以金和银纳米粒子为代表的贵金属纳米粒子 有着特殊的光学性质, 表现出许多常规块体材料所 不具备的优异性能, 其中局域表面等离激元共振 (LSPR)特性是研究热点之一. LSPR是一种物理光学 现象, 当一定频率的入射光与比其波长尺寸小的金 属纳米粒子作用, 入射光子与纳米粒子的等离子体 在粒子周边所发生的局域共振. ${ }^{1}$ 具有 LSPR特性的 金属纳米粒子的大小、形状以及周围介质折射率 都可能影响其LSPR的最大吸收峰位 $\left(\lambda_{\text {max }}\right)$ 和峰形状, 研究者利用这些性质已发展了不少化学生物传感 器..$^{2-4}$ 当金属纳米粒子与金膜相互靠近达到纳米间 隔时, 在合适的激发光频率和偏振光状态下, 粒子 与金膜之间结合部位的局域电磁场由于近场耦合 效应而得到极为显著的增强, 增强倍数甚至可达到 百万倍. ${ }^{5}$

人们结合扫描探针技术和贵金属的LSPR效应 发展了针尖增强拉曼光谱(TERS)技术, 这种技术既 具有较高的空间分辨率, 又能得到很强的谱学信号, 我们研究组在这方面做出了一些开拓性的工作. 近 来, 我们又试图拓展研究范围, 将研究焦点从谱学 传感转移到定位、测距上, 利用贵金属纳米探针与 基底耦合结构产生的LSPR会随着环境折射率及耦 合距离变化发生峰 $\left(\lambda_{\max }\right)$ 位移, 发展一种高空间分辨 的探针技术——“纳米间隙耦合增强表面等离激元 共振扫描显微技术”. ${ }^{6}$ 这一技术的关键是制备光纤 探针, 须将合成的具有高介电敏感性的纳米粒子吸 附在光纤的末端, 再由光纤引入入射光激发纳米粒 子产生LSPR. 虽然现有研究表明一些非球形的纳米 粒子较球形粒子具有更多电磁场“热点”, 从而对其 周围环境折射率的变化更加敏感? 但纳米粒子在实 际应用于光纤探针时, 由于光纤探针末端上修饰纳 米粒子的取向控制和激发光入射角度限制, 光纤引 入的激发光往往难以有效激发非球形纳米粒子的 热点, 所以在本文中的探针制备采用对激发光角度 无选择性的球形纳米粒子. 8.9 接下来需要解决的关 键问题就是如何提高修饰于针尖末端的球形纳米
粒子LSPR信号的介电敏感性. 对于贵金属纳米粒 子, 具有较强 LSPR信号的尺寸范围约为 10-200 $\mathrm{nm} .{ }^{10}$ 当纳米粒子粒径较大时, 散射更强, 有利于提 高信噪比, 但会出现四极峰, 导致半峰宽变宽, ${ }^{11,12}$ 降 低峰检测的分辨率; 而纳米粒子粒径较小时, 有利 于提高检测的空间分辨率, 但纳米粒子散射较弱. ${ }^{13}$ 我们所发展的光纤探针技术需要兼顾高空间分辨 和高信噪比, 选择合适大小的纳米粒子十分重要. 不同材料损耗性质 $Q$ 因子(材料介电实部/虚部)越大, 其SPR损耗越小, 对折射率越敏感. $\mathrm{Ag}$ 的 $Q$ 因子约为 80-100, $\mathrm{Au}$ 的 $Q$ 因子约为 10-20, 其他材料均小于 $\mathrm{Au}$ 和 $\mathrm{Ag},{ }^{14,15}$ 所以同样形状尺寸下银纳米粒子往往 比金纳米粒子有着更高的介电敏感性. ${ }^{16}$ 除Chumanov 等 ${ }^{2}$ 利用氢气还原 $70{ }^{\circ} \mathrm{C}$ 下过饱和的 $\mathrm{AgO}_{2}$ 溶液可得 到尺寸较大的银纳米球的方法外(但此方法较繁琐), 现有文献报道的银纳米粒子的合成方法可以得到 较小尺寸 $(<40 \mathrm{~nm})$ 、均一性好的球形纳米粒子, ${ }^{17,18}$ 但在合成较大尺寸 $(>40 \mathrm{~nm})$ 的球形纳米粒子时, 就 难于保持较好的均一性. ${ }^{19,20}$ 为了方便得到较大尺寸 的均一性好的纳米粒子, 本文采用的策略是以 $\mathrm{Au}$ 纳 米粒子为核, 再包上一层较厚的 $\mathrm{Ag}$ 壳层. 这样既能 得到我们所需大小的均一性好的纳米粒子, 又能使 纳米粒子主要体现为 $\mathrm{Ag}$ 的性质而具有较高的介电 敏感性. 考虑到 $\mathrm{Ag}$ 纳米粒子大于 $70 \mathrm{~nm}$ 时会出现四 极峰, ${ }^{12}$ 本文选用尺寸为 $70 \mathrm{~nm}$ 的纳米粒子. 我们采 用Xia等 ${ }^{18}$ 提出的阴离子辅助水相法, 在不同尺寸的 $\mathrm{Au}$ 核上包覆不同厚度的 $\mathrm{Ag}$ 壳层, 得到核壳比不同、 均一性好的 $\mathrm{Au} @ \mathrm{Ag}$ 纳米粒子. 研究与其紧密接触的 不同折射率介质对其LSPR信号的影响, 探索用于光 纤探针的纳米粒子的结构和合成方法.

\section{2 实验部分}

\section{1 试剂与仪器}

氯金酸 $\left(\mathrm{HAuCl}_{4} \cdot 4 \mathrm{H}_{2} \mathrm{O}, \geq 99.0 \%\right)$, 抗坏血酸( $\mathrm{AA}, \geq$ 99.7\%), 柠檬酸三钠 $\left(\mathrm{Na}_{3} \mathrm{C}_{6} \mathrm{H}_{5} \mathrm{O}_{7} \cdot 2 \mathrm{H}_{2} \mathrm{O}, \geq 99.0 \%\right)$, 硝 酸银 $\left(\mathrm{AgNO}_{3}, \geq 99.8 \%\right)$, 浓盐酸 $(\mathrm{HCl}, 36 \%-38 \%)$, 浓 硝酸 $\left(\mathrm{HNO}_{3}, 65 \%-68 \%\right)$, 浓硫酸 $\left(\mathrm{H}_{2} \mathrm{SO}_{4}, 95 \%-98 \%\right)$, 
双氧水 $\left(\mathrm{H}_{2} \mathrm{O}_{2}, \geq 30 \%\right)$ 和无水碳酸钠 $\left(\mathrm{Na}_{2} \mathrm{CO}_{3}, \geq\right.$ $99.8 \%$ )均购自国药集团化学试剂有限公司, 所有的 化学试剂没有经过进一步的提纯. 所有的玻璃仪器 经过王水 $\left(3: 1(V / V) \mathrm{HCl}(37 \%) / \mathrm{HNO}_{3}(65 \%)\right)$ 和浓硫 酸双氧水 $\left(3: 1(V / V) \mathrm{H}_{2} \mathrm{SO}_{4}(98 \%) / \mathrm{H}_{2} \mathrm{O}_{2}(30 \%)\right)$ 浸泡， 再用去离子水反复冲洗. 实验用水均采用 Millipore公司超纯水仪提供的电阻率大于 18.2 $\mathrm{M} \Omega \cdot \mathrm{cm}$ 的超纯水.

日本日立公司生产的Hitachi S-4800场发射扫 描电子显微镜 (SEM) 和荷兰FEI公司生产的 300 $\mathrm{kV}$ 高分辨Tecnai F30透射电子显微镜(TEM)用于表 征纳米粒子的尺寸及形貌特征; 美国Varian公司生 产的Carry-5000紫外-可见-近红外分光光度计用于 测试纳米粒子的紫外-可见消光谱; 暗场显微镜 (Leica DMI 3000, 德国), 共聚焦拉曼光谱仪Renishaw inVia (英国Renishaw公司)用于采集单颗纳米粒子 的散射光谱.

\section{$2.2 \mathrm{Au} @ \mathrm{Ag}$ 纳米粒子的合成}

\subsubsection{Au核纳米粒子的合成}

采用Frens ${ }^{21}$ 的方法合成 $15 \mathrm{~nm}$ 的 $\mathrm{Au}$ 纳米粒子. 取 $1.21 \mathrm{~mL} \mathrm{HAuCl}_{4}(1 \%)$ 配制成 $100 \mathrm{~mL}$, 溶液在剧烈 搅拌下加热至沸腾, 迅速加入 $4 \mathrm{~mL} 1 \%$ 的柠檬酸钠 水溶液, 持续煮沸 $40 \mathrm{~min}$ 后自然冷却搅拌至室温.

采用Ziegler和Eychmüller ${ }^{11}$ 的种子生长法合成 32 和 $52 \mathrm{~nm}$ 的 $\mathrm{Au}$ 纳米粒子. 合成 $32 \mathrm{~nm}$ 的 $\mathrm{Au}$ 纳米粒 子: 将 $3 \mathrm{~mL} 15 \mathrm{~nm} \mathrm{Au}$ 种在双口烧瓶中稀释到 $20 \mathrm{~mL}$, $\mathrm{A}$ 液(10 mL含有 $0.48 \mathrm{~mL} \mathrm{HAuCl}{ }_{4}(1 \%)$ 的水溶液)和 $\mathrm{B}$ 液 $(10 \mathrm{~mL}$ 含有 $0.60 \mathrm{~mL}$ 柠檬酸三钠 $(1 \%)$ 和 0.30 $\mathrm{mL}$ 抗坏血酸 $(1 \%)$ 的水溶液)通过双通道步进机 40 $\min$ 同时加入到 $\mathrm{Au}$ 种中摚拌反应, 加液完成后直接 将混合物升温至沸腾并搅拌, 并保持此温度 $30 \mathrm{~min}$, 最后自然冷却搅拌至室温. 合成 $52 \mathrm{~nm}$ 的 $\mathrm{Au}$ 纳米粒 子: 将冷却的 $16.1 \mathrm{~mL}, 32 \mathrm{~nm} \mathrm{Au}$ 种在双口烧瓶中稀 释到 $20 \mathrm{~mL}$, 将上述 $\mathrm{A}$ 液和B液通过双通道步进机 40 $\min$ 同时加入上述溶液中, 并不断搅拌, 接下来回流 加热30 min后自然冷却至室温.

\subsubsection{Au@Ag核壳纳米粒子的合成}

将 $10 \mathrm{~mL}$ 含有 $215 \mu \mathrm{L}$ 柠檬酸三钠( $1 \%), 310 \mu \mathrm{L}$, $\mathrm{AgNO}_{3}\left(10 \mathrm{mmol} \cdot \mathrm{L}^{-1}\right)$ 和 $15 \mu \mathrm{L}, \mathrm{Na}_{2} \mathrm{CO}_{3}(0.05 \mathrm{mmol} \cdot$ $\left.\mathrm{L}^{-1}\right)$ 的水溶液在室温下摚拌 $5 \mathrm{~min}$, 将其通过步进机 分别加入到 $20 \mathrm{~mL}$ 含 $105 \mu \mathrm{L}, 15 \mathrm{~nm} \mathrm{Au}$ 核, $20 \mathrm{~mL}$ 含 $1.5 \mathrm{~mL}, 32 \mathrm{~nm} \mathrm{Au}$ 核和 $30 \mathrm{~mL}$ 含 $5.5 \mathrm{~mL}, 52 \mathrm{~nm} \mathrm{Au}$ 核 的溶液中, $40 \mathrm{~min}$ 加完. 最后回流摚拌加热 $30 \mathrm{~min}$,
自然冷却至室温, 分别得到三种不同 $\mathrm{Ag}$ 壳层厚度的 $\mathrm{Au} @ \mathrm{Ag}$ 纳米粒子.

\section{3 纳米粒子溶液在折射率不同的溶液中的介电 敏感性检测}

测量粒子分散在不同的折射率溶液中的消光 谱时, 先将纳米粒子离心清洗, 转速为 $3000 \mathrm{r} \cdot \mathrm{min}^{-1}$, 清洗两次，再将粒子分别分散在超纯水、 $20 \%$ 、 $30 \% 、 40 \% 、 50 \% 、 60 \%(w)$ 的葡萄糖溶液中, 进行 紫外-可见消光谱的测量.

\section{4 单颗纳米粒子的LSPR测量}

首先将 $50 \mu \mathrm{L} \mathrm{Au} @ \mathrm{Ag} \mathrm{NPs}$ 的溶液稀释到1 $\mathrm{mL}$ 的去离子水中, 接下来将其分别滴在二氧化硅 $\left(\mathrm{SiO}_{2}\right)$ 、三氧化二铝 $\left(\mathrm{Al}_{2} \mathrm{O}_{3}\right)$ 、二氧化钛 $\left(\mathrm{TiO}_{2}\right)$ 的非导 电性基底上, 基底都含有通过光刻加工的定位网格, 再通过真空洜将水分抽干完成基底的制备.

暗场显微镜能使光从一定的角度入射, 纳米粒 子的散射光通过一个更小的角度收集 (由镜头的数 值孔径(NA) 决定), 本实验采用的暗场镜头为 50 倍 NA 0.55 的镜头. 我们课题组在商品化的Renishaw inVia共聚焦拉曼光谱仪的基础上, 研制了正置倒置 联用的暗场光谱采集系统, 建立了暗场和扫描电镜 共定位的方法, 实现同一颗纳米粒子的暗场光谱表 征和电镜形貌表征.

\section{3 结果与讨论}

\section{$3.1 \mathrm{Au} @ A g$ 纳米粒子的表征}

采用颈基硅烷分子作为链接分子将溶液中的 单颗纳米粒子修饰到光纤探针的末端. ${ }^{22}$ 这种单颗 粒子的自组装方法虽然较为简便, 但对不同尺寸的 纳米粒子不具选择性, 为了将设定尺寸的纳米粒子 修饰上去, 就要求溶液中的纳米粒子具有较好的尺 寸均一性. 制备尺寸均一的 $\mathrm{Au} @ \mathrm{Ag}$ 纳米粒子, 首先 需要保证作为核的纳米结构足够均匀. 我们制备了 三种尺寸分别为 $15,32,52 \mathrm{~nm}$ 的 $\mathrm{Au}$ 纳米粒子. 如图 $1(\mathrm{a}, \mathrm{b}, \mathrm{c})$ 的扫描电镜图所示, 所制备的三种不同直 径 $(15,32,52 \mathrm{~nm})$ 的Au纳米粒子尺寸分布较为均一, 图 $1 \mathrm{~d}$ 为三种直径的 $\mathrm{Au}$ 纳米粒子的紫外-可见消光光 谱, 三种纳米粒子的峰位置分别为 513、523、532 $\mathrm{nm}$, 半峰宽分别为 $68 、 64 、 71 \mathrm{~nm}$. 随着纳米粒子直 径的增大, 峰位置有所红移, 半峰宽与Ziegler和 Eychmüller ${ }^{11}$ 合成的相应尺寸的 $\mathrm{Au}$ 纳米粒子半峰宽 相当, 进一步说明合成的三种 $\mathrm{Au}$ 纳米粒子尺寸分布 较窄, 粒径较为均一.

在大多数制备 $\mathrm{Au} @ \mathrm{Ag}$ 纳米核壳粒子的研究中, 

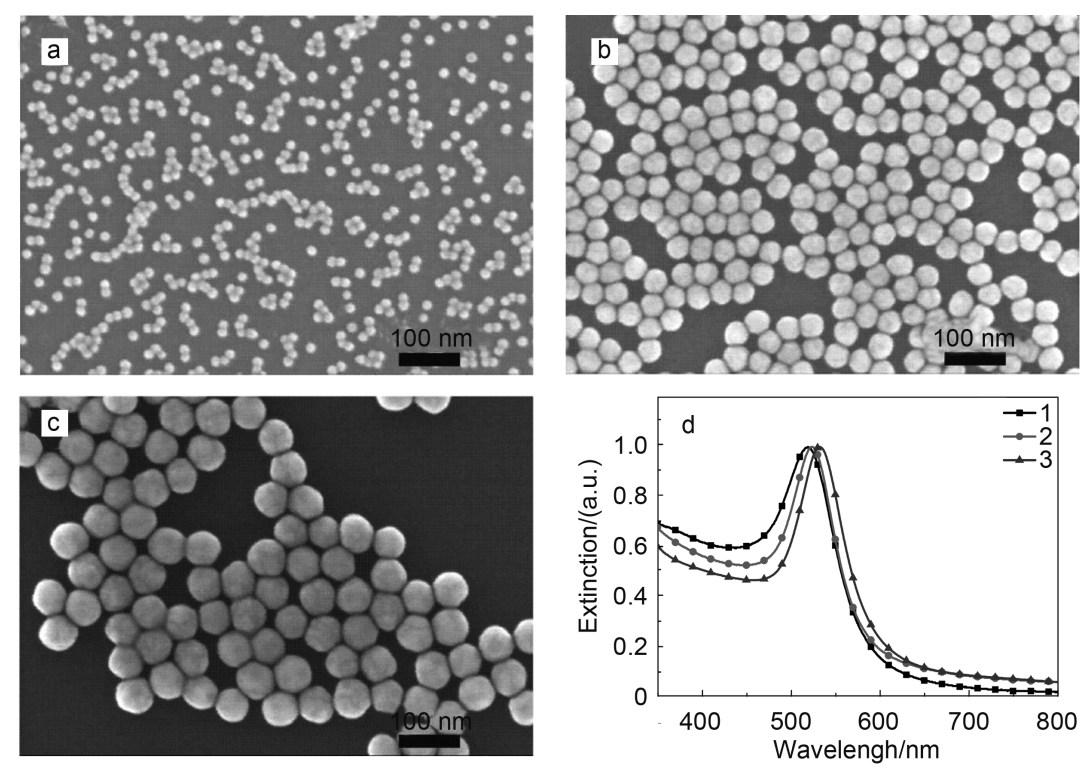

图1 不同粒径的Au核的扫描电镜(SEM)形貌图和紫外-可见消光光谱图

Fig.1 Scanning electron microscope (SEM) images and UV-Vis extinction spectra of different particle sizes of Au core

particle size/nm: (a) $15.4 \pm 1.9$; (b) $32.1 \pm 2.8$; (c) $51.6 \pm 4.3$; in the spectra of (d), particle size/nm: (1) $15.4 \pm 1.9$; (2) $32.1 \pm 2.8$; (3) $51.6 \pm 4.3$

采用不同的还原剂控制 $\mathrm{Ag}^{+}$在 $\mathrm{Au}$ 纳米粒子表面形成 壳层的成核速度, 得到的 $\mathrm{Ag}$ 壳层相对较薄, 约为 1-10 nm. ${ }^{23-26}$ 虽然Pastoriza-Santos 等 ${ }^{27}$ 报道过较厚 $\mathrm{Ag}$ 壳层的 $\mathrm{Au} @ \mathrm{Ag}$ 纳米颗粒的合成, 但需要多次包 覆, 耗时较长. $\mathrm{Xia}$ 等 ${ }^{18}$ 利用 $\mathrm{Cl}^{-} 、 \mathrm{Br} 、 \mathrm{I} 、 \mathrm{SO}_{4}^{2-} 、 \mathrm{CO}_{3}^{2-}$ 、 $\mathrm{PO}_{4}^{3-}$ 、和 $\mathrm{S}^{2-}$ 等阴离子与 $\mathrm{Ag}^{+}$的相互作用, 在溶液中 加入这些阴离子形成 $\mathrm{Ag}$ 的前驱体从而改变 $\mathrm{Ag}^{+}$的还 原电势, 更好地控制 $\mathrm{Ag}^{+}$的还原速度, 合成了尺寸均 一、直径为 $23-30 \mathrm{~nm}$ 的 $\mathrm{Ag}$ 纳米粒子. 本文首次将此 法引入到 $\mathrm{Au} @ \mathrm{Ag}$ 纳米粒子的合成中, 在 $\mathrm{Au}$ 核上一 步合成较厚的 $\mathrm{Ag}$ 壳层. 在本实验中采用了煮沸后易 于挥发的 $\mathrm{CO}_{3}^{2-}$ 作为辅助的阴离子. 通过设计 $\mathrm{Ag}$ 壳层 的厚度, 控制 $\mathrm{Au}$ 核与 $\mathrm{Ag}^{+}$的量之比来实现不同厚度 的 $\mathrm{Ag}$ 壳层包覆, 从而获得外径大概为 $70 \mathrm{~nm}$ 、核壳 比不同的 $\mathrm{Au} @ \mathrm{Ag}$ 纳米粒子. 由图 $2(\mathrm{a}, \mathrm{b}, \mathrm{c})$ 的SEM图 可知, 所合成的三种核壳比不同的 $\mathrm{Au} @ \mathrm{Ag}$ 纳米粒子 粒径分布较为均一. 我们根据SEM图(图1和图2)统 计了核壳比不同的三种比例的 $\mathrm{Au} @ \mathrm{Ag}$ 纳米粒子的 直径、 $\mathrm{Au}$ 核直径, 以及将 $\mathrm{Au} @ \mathrm{Ag}$ 纳米粒子的平均 直径减去 $\mathrm{Au}$ 核的平均直径后得到的 $\mathrm{Ag}$ 壳层的平均 厚度, 详细数据见表 1 . 从表 1 中可知三种核壳比的 $\mathrm{Au} @ \mathrm{Ag}$ 纳米粒子尺寸的相对标准偏差都小于 $10 \%$. 从 TEM图(图2(d,e, f))中可清晰地观察到 $26 \mathrm{~nm}$ $\mathrm{Au} @ 10 \mathrm{~nm} \mathrm{Ag}$ 纳米粒子的核壳结构. 对于7.5 nm

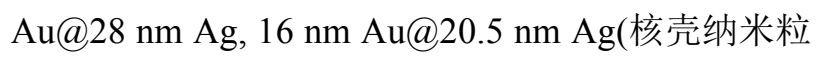

子的表示方法皆为: 核的半径@壳层厚度, 下文全 为该种表述方法)这两种纳米粒子, 由于 $\mathrm{Ag}$ 壳层太 厚 $(\geq 20 \mathrm{~nm}$ ), 难以清晰的观察到核壳结构. 为了进 一步确定三种比例的 $\mathrm{Au} @ \mathrm{Ag}$ 纳米粒子的核壳结构, 我们使用了扫描透射电子显微镜(STEM)中X射线 能谱线扫描进行原位分析. 图 $2(\mathrm{~g}, \mathrm{~h}, \mathrm{j})$ 分别是三种比 例的 $\mathrm{Au} @ \mathrm{Ag}$ 纳米粒子元素含量变化的分布曲线, 插 图是对应扫描的纳米粒子的暗场图像. 从插图中暗 场图像可较为清晰地看到三种粒子的核壳结构. 根 据X射线能谱线扫描曲线获得的三种核壳比的 $\mathrm{Au} @ \mathrm{Ag}$ 纳米粒子核、壳尺寸与电镜图测量的数值 也是相吻合的. 我们将加入的 $\mathrm{Ag}^{+}$物质的量换算为 $\mathrm{Au}$ 核上包覆的 $\mathrm{Ag}$ 壳层得到 $\mathrm{Au} @ \mathrm{Ag}$ 的直径(表1的第 4列), 计算得到的数值与电镜实验测量值在实验误 差范围内也是一致的. 此外, 无论是在SEM图还是 TEM图中都没有发现在 $\mathrm{Au} @ \mathrm{Ag}$ 附近有 $\mathrm{Ag}$ 的自生成 核. 也就是说, 加入的 $\mathrm{Ag}$ 前驱体基本上都成为包覆 $\mathrm{Au}$ 核的 $\mathrm{Ag}$ 壳层. 这表明该方法在保证核壳结构纳米 粒子尺寸均一性的同时, 能较为可控地、容易地一 步获得较厚 $(\geq 20 \mathrm{~nm})$ 的 $\mathrm{Ag}$ 壳层.

图3 是三种比例的 $\mathrm{Au} @ \mathrm{Ag}$ 纳米粒子的紫外-可 见消光光谱图(光强度经归一化处理). 相比于 $A u$ 纳 米粒子溶液消光谱峰中只含有 $\mathrm{Au}$ 的 LSPR峰, $\mathrm{Au} @ \mathrm{Ag}$ 纳米粒子在 $400 \mathrm{~nm}$ 附近会出现一个银的 LSPR 峰. 对于 $\mathrm{Ag}$ 壳层较厚的 $\mathrm{Au} @ \mathrm{Ag}$ 纳米粒子, 由 

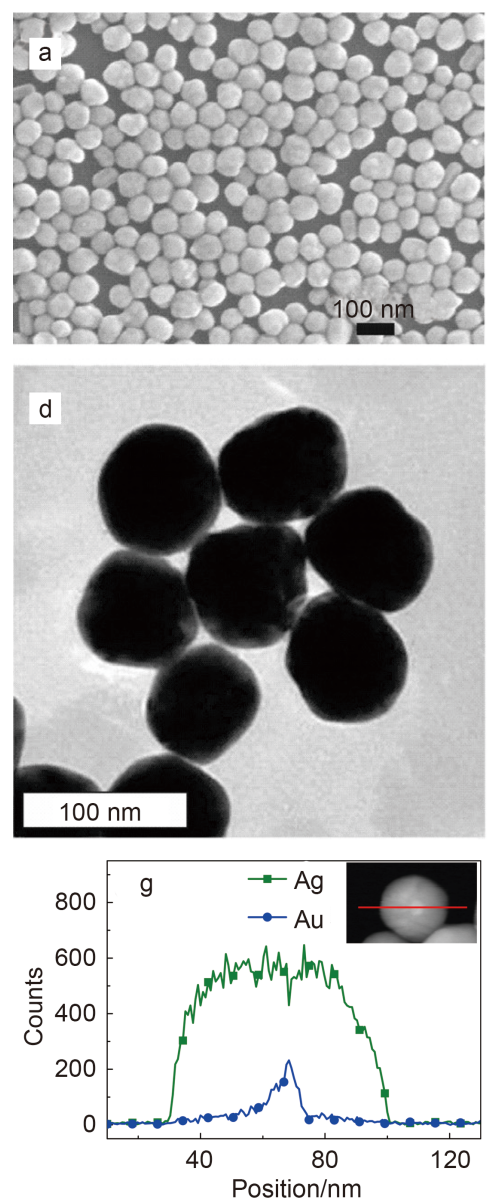
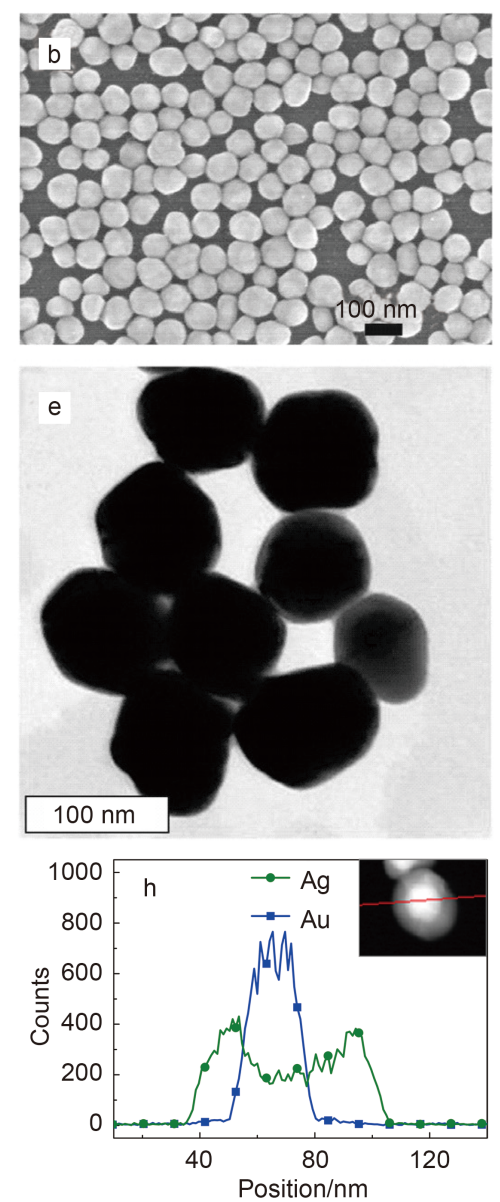
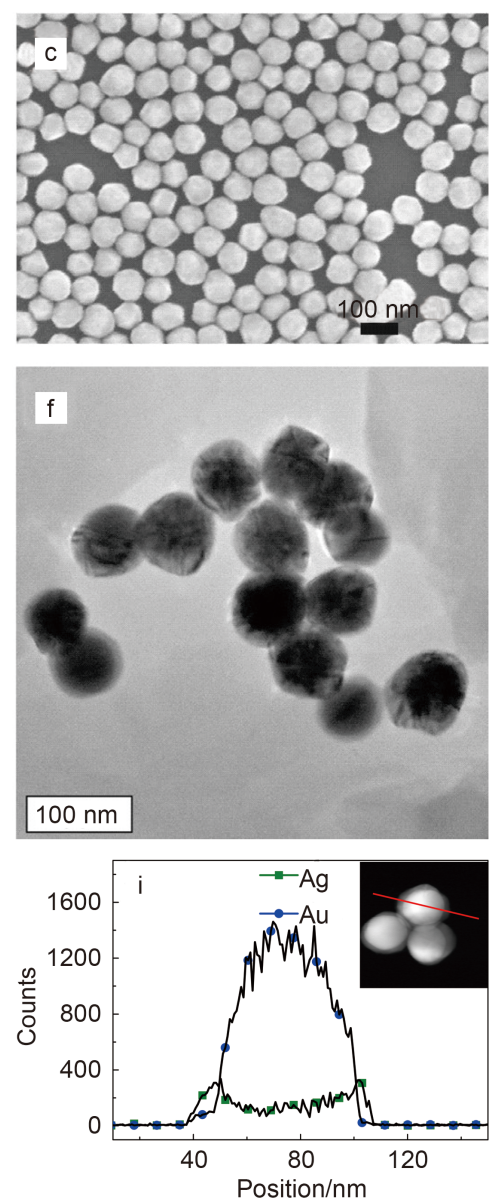

图2 不同核壳比的Au@Ag纳米粒子的SEM形貌图(a-c)、透射电镜(TEM)形貌图(d-f)及扫描透射电子显微镜(STEM)X射线 能谱(EDS)线扫描分析 $(\mathrm{g}-\mathrm{i})$

Fig.2 SEM images (a-c), TEM images (d-f), and scanning transmission electron microscopy-energy dispersive $\mathrm{X}$-ray spectroscopy (STEM-EDS) line scanning analyses (g-i) of different core-shell ratio of Au@Ag NPs

(a, d, g) 7.5 nm Au@28 nm Ag; (b, e, h) 16 nm Au@20.5 nm Ag; (c, f, i) 26 nm Au@10 nm Ag

表1 Au@Ag纳米粒子尺寸细节

Table 1 Details of the sizes of Au@Ag NPs

\begin{tabular}{cccc}
\hline $\begin{array}{c}\text { Au@Ag NPs with different } \\
\text { core-shell ratio }\end{array}$ & $\begin{array}{c}\text { Diameter of Au } \\
\text { core/nm }\end{array}$ & $\begin{array}{c}\text { Diameter of Au@Ag } \\
\text { NPs/nm }\end{array}$ & $\begin{array}{c}\text { Diameter of Au@Ag NPs by } \\
\text { calculation/nm }\end{array}$ \\
\hline $7.5 \mathrm{~nm} \mathrm{Au} @ 28 \mathrm{~nm} \mathrm{Ag}$ & $15.4 \pm 1.9$ & $70.6 \pm 6.7(9 \%)^{*}$ & 70.1 \\
$16 \mathrm{~nm} \mathrm{Au} @ 20.5 \mathrm{~nm} \mathrm{Ag}$ & $32.1 \pm 2.8$ & $72.7 \pm 5.6(8 \%)^{*}$ & 70.2 \\
$26 \mathrm{~nm} \mathrm{Au@10} \mathrm{nm} \mathrm{Ag}$ & $51.6 \pm 4.3$ & $71.5 \pm 4.8(7 \%)^{*}$ & 69.8 \\
\hline
\end{tabular}

"Values in brackets represent the standard deviation.

于 $\mathrm{Ag}$ 层的吸收, $520 \mathrm{~nm}$ 左右的入射光衰减严重, 内 核Au的LSPR无法被有效激发, 使得Au的信号无法 被检测, 如图3中7.5 nm Au@28 nm Ag, 16 nm $\mathrm{Au} @ 20.5 \mathrm{~nm} \mathrm{Ag}$ 这两种纳米粒子的消光谱峰. 而对 于银层较薄的 $\mathrm{Au} @ \mathrm{Ag}$ 纳米粒子, 可以观测到 $\mathrm{Au}$ 的 LSPR峰, 如图3中 $26 \mathrm{~nm} \mathrm{Au} @ 10 \mathrm{~nm} \mathrm{Ag}$ 纳米粒子的

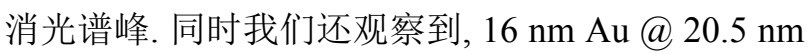
$\mathrm{Ag}$ 纳米粒子在 $400 \mathrm{~nm}$ 处可以观察到一个很小的四 极峰. ${ }^{27}$ 从消光谱中我们还可以看到7.5 nm Au@28 $\mathrm{nm} \mathrm{Ag}$ 的峰位置为 $427 \mathrm{~nm}$, 与米氏理论计算得到的 $70 \mathrm{~nm}$ 的 $\mathrm{Ag}$ 纳米粒子在水溶液中的消光谱(图3曲线 4)峰位置能较好地重合. 理论计算曲线在 $375 \mathrm{~nm}$ 处 的峰为四极峰. 说明这种方法合成较厚 $\mathrm{Ag}$ 壳层的 $\mathrm{Au} @ \mathrm{Ag}$ 纳米粒子, 能使其光学性质与相同大小的 $\mathrm{Ag}$ 纳米粒子的光学性质相似.

\section{2 不同核壳比Au@Ag纳米粒子在不同折射率 溶液中的介电敏感性}

我们研究组提出的纳米间隙耦合增强表面等 


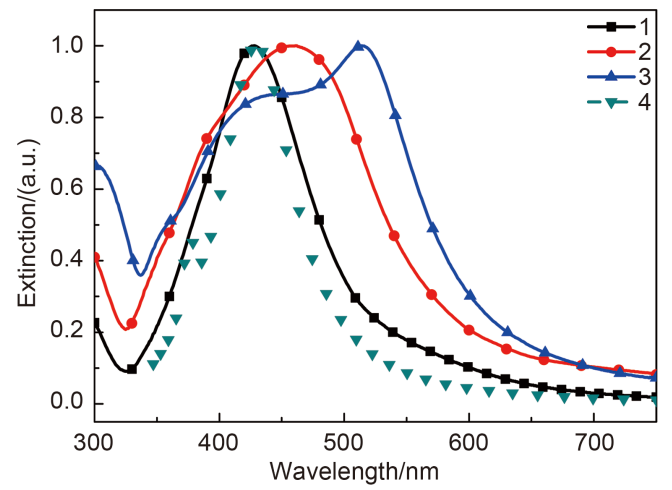

图3 不同核壳比的 $\mathrm{Au} @ \mathbf{A g}$ 纳米粒子的紫外-可见消光光谱 图和计算(点线)得到的 $70 \mathrm{~nm}$ 的 $\mathrm{Ag}$ 纳米粒子的消光光谱图

Fig.3 UV-Vis extinction spectra of Au@Ag NPs with different core-shell ratios and calculated (dotted lines) spectra of $70 \mathrm{~nm} \mathrm{Ag} \mathrm{NPs}$

(1) 7.5 nm Au@28 nm Ag; (2) 16 nm Au@20.5 nm Ag; (3) 26 nm Au@10 $\mathrm{nm} \mathrm{Ag}$; (4) $70 \mathrm{~nm} \mathrm{Ag}$ (the peak of $375 \mathrm{~nm}$ is a quadrupole peak.)

离激元共振扫描显微技术希望应用于小至 1-10 $\mathrm{nm}$ 的纳米尺度的表界面研究, ${ }^{6}$ 因此有必要探究具 有高介电敏感性的纳米粒子结构. 文献中评估金属 纳米粒子的LSPR介电敏感性时一般有两种测量方 式: 一种是将纳米粒子溶液离心后置于不同折射率 的溶液中测量其LSPR峰位移, 得到的是溶液中大量 纳米粒子的整体效应; 另一种是将单颗纳米粒子置 于不同折射率的溶液中，测量单颗纳米粒子的 LSPR峰位移.2溶液中纳米粒子整体测量较为简便, 但由于粒子尺寸分布、粒子团聚、内核偏离中心 等原因导致其测得的半峰宽大于单颗纳米粒子测 得的半峰宽, 而半峰宽较窄有利于介电敏感性的检 测. 首先将纳米粒子溶液离心后置于不同折射率的 葡萄糖溶液中, 考察三种不同核壳比 $\mathrm{Au} @ \mathrm{Ag}$ 纳米粒 子溶液在不同折射率溶液中的介电敏感性.
如图4所示, 三种不同核壳比例的 $\mathrm{Au} @ \mathrm{Ag}$ 纳米 粒子在不同折射率的溶液 (折射率分别为 1.333 、 $1.364 、 1.381 、 1.399 、 1.418 、 1.439)$ 中都产生了位 移. 由图 5 及表 2 折射率与位移关系曲线可知, 三种 纳米粒子的介电敏感性分别为219( $( \pm 7) 、 224( \pm 9)$ 和105 $( \pm 10) \mathrm{nm} \cdot \mathrm{RIU}^{-1}$ (RIU: refractive index unit). 已 有文献报道单颗 $60 \mathrm{~nm}$ 的 $\mathrm{Ag}$ 纳米球的介电敏感性为 $160 \mathrm{~nm} \cdot \mathrm{RIU}^{-1},{ }^{28} 50 \mathrm{~nm}$ 的 $\mathrm{Au}$ 纳米球溶液的介电敏感 性为 $60 \mathrm{~nm} \cdot \mathrm{RIU}^{-1} \cdot{ }^{29} \mathrm{Ag}$ 壳层较薄的 $26 \mathrm{~nm} \mathrm{Au@10} \mathrm{nm}$ $\mathrm{Ag}$ 纳米粒子介电敏感性虽然比 $60 \mathrm{~nm}$ 的 $\mathrm{Ag}$ 纳米球 要低, 却远大于 $50 \mathrm{~nm}$ 的 $\mathrm{Au}$ 纳米球. $26 \mathrm{~nm} \mathrm{Au@10}$ $\mathrm{nm} \mathrm{Ag}$ 纳米粒子的介电敏感性较小, 这可能是因为 其 $\mathrm{Ag}$ 壳层较薄, 更多的还是以 $\mathrm{Au}$ 的性质为主. 对于 $\mathrm{Ag}$ 壳层较厚的7.5 nm Au@28 nm Ag, 16 nm Au@ $20.5 \mathrm{~nm} \mathrm{Ag}$ 这两种纳米粒子在溶液中测得的介电敏 感性要优于单颗 $60 \mathrm{~nm}$ 的 $\mathrm{Ag}$ 纳米球的介电敏感性. 同时7.5 nm Au@28 nm Ag, 16 nm Au@20.5 nm $\mathrm{Ag}$ 这两种纳米粒子的单位折射率位移大小基本相 同(在实验误差范围内), 表明这两种纳米粒子的 $\mathrm{Ag}$ 壳层厚到一定程度 $(\geq 20 \mathrm{~nm})$, 主要以 $\mathrm{Ag}$ 的 LSPR性质为主.

在研究LSPR介质敏感性时常采用单位折射率 的光谱峰位移来定量评估. 然而, 尺寸大的纳米粒 子虽往往有较高的介电敏感性, 但由于辐射损耗较 大使它们的LSPR半峰宽变大. 这种情况下将单位折 射率的光谱峰位移除以半峰宽, 得到所谓的品质因 子(FOM), 可进一步用以衡量纳米粒子的介质传感 能力. ${ }^{30}$ 由表 2 可知, 三种纳米粒子的FOM值分别为 2.3、1.4、0.6. 对于7.5 nm Au@28 nm Ag纳米粒子, 其FOM值比文献中单颗 $60 \mathrm{~nm} \mathrm{Ag}$ /纳米球的FOM值

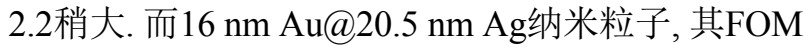
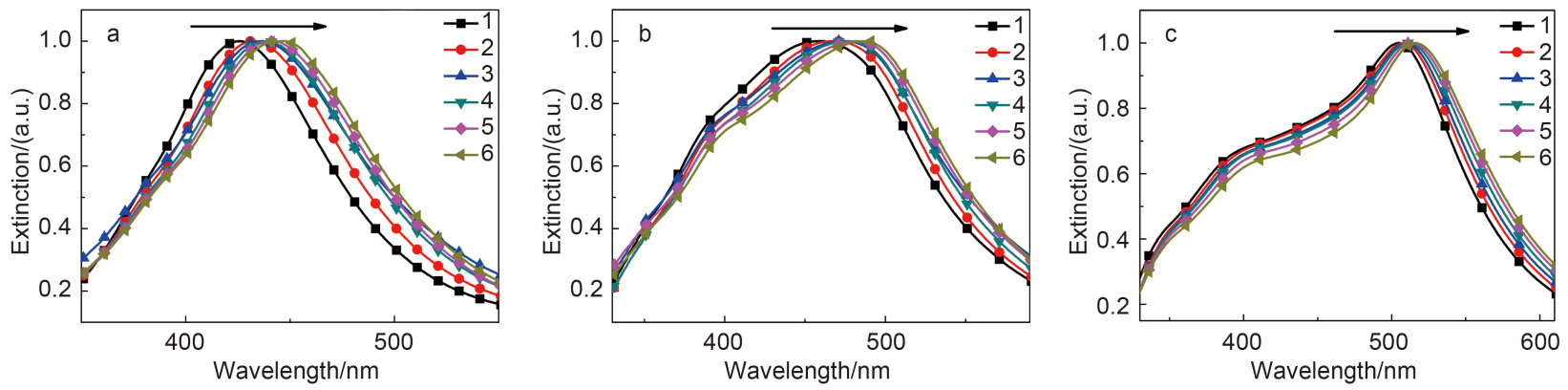

图4 不同核壳比的Au@Ag纳米粒子在不同折射率的溶液中的位移

Fig.4 Shift caused by different core-shell ratios of $\mathrm{Au} @ \mathrm{Ag}$ NPs in the solution with different refractive indexes

(a) 7.5 nm Au@28 nm Ag; (b) 16 nm Au@20.5 nm Ag; (c) 26 nm Au@10 nm Ag; the refractive index successively increases in the direction of arrow: (1) 1.333, (2) 1.364 , (3) 1.381, (4) 1.399, (5) 1.418, , (6) 1.439 


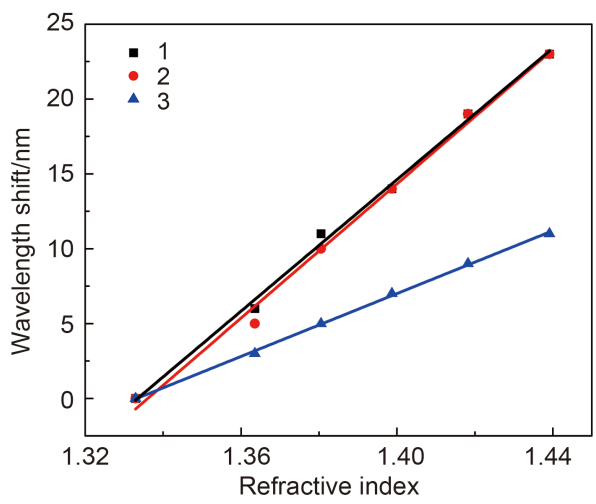

图5 不同核壳比的 $\mathrm{Au} @ \mathbf{A g}$ 纳米粒子的峰位移与折射率的 线性关系

Fig.5 Linear relationship between peak shift of $\mathrm{Au} @ \mathrm{Ag}$ nanoparticles with different core-shell ratios and refractive index

(1) 7.5 nm Au@28 nm Ag; (2) 16 nm Au@ 20.5 nm Ag; (3) $26 \mathrm{~nm} \mathrm{Au@10} \mathrm{nm} \mathrm{Ag}$

值比单颗 $60 \mathrm{~nm} \mathrm{Ag}$ 纳米球低, 主要由于文献中采用 的是单颗纳米粒子进行测量 (半峰宽大概为 $70 \mathrm{~nm}$ ), 而我们采用的是整体纳米粒子溶液进行测量(半峰 宽大至 $155 \mathrm{~nm})$, 两种测量方法的半峰宽有很大不 同, 故计算所得到的FOM值小于文献中 $60 \mathrm{~nm} \mathrm{Ag}$ 纳

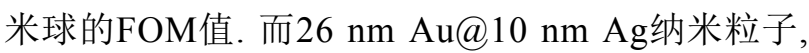
$\mathrm{Au}$ 和 $\mathrm{Ag}$ 的性质在其消光谱中同时存在, 半峰宽较大 (大概为 $190 \mathrm{~nm}$ ), 其FOM值比 $50 \mathrm{~nm} \mathrm{Au}$ 纳米球溶液 测得的FOM值 1.5 小. 对于这三种核壳比不同的 $\mathrm{Au} @ \mathrm{Ag}$ 纳米粒子, 其FOM值存在7.5 nm Au@28 nm

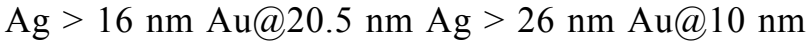
$\mathrm{Ag}$ 的关系. 由三种纳米粒子单位折射率位移大小和 FOM值的比较结果, 可知7.5 nm Au@28 nm Ag纳米 粒子更适合用于传感.
表2 根据图5计算得到的不同核壳比的 $\mathrm{Au} @ \mathbf{A g}$ 纳米粒子的 介电敏感性和FOM值

Table 2 Dielectric sensitivity and FOM values of $\mathrm{Au} @ \mathrm{Ag}$ NPs with different core-shell ratios calculated according to Fig.5

\begin{tabular}{ccc}
\hline $\begin{array}{c}\text { Au@Ag NPs with different } \\
\text { core-shell ratios }\end{array}$ & Sensitivity/(nm·RIU $\left.{ }^{-1}\right)$ & FOM \\
\hline $7.5 \mathrm{~nm} \mathrm{Au} @ 28 \mathrm{~nm} \mathrm{Ag}$ & $219 \pm 7$ & 2.3 \\
$16 \mathrm{~nm} \mathrm{Au} @ 20.5 \mathrm{~nm} \mathrm{Ag}$ & $224 \pm 9$ & 1.4 \\
$26 \mathrm{~nm} \mathrm{Au} @ 10 \mathrm{~nm} \mathrm{Ag}$ & $105 \pm 10$ & 0.6 \\
\hline
\end{tabular}
RIU: refractive index unit; FOM: figure of merit

\section{3 不同核壳比Au@Ag纳米粒子在折射率不同} 的非导电性基底上的散射光谱研究

我们所发展的纳米间隙耦合增强表面等离激 元共振扫描显微技术, 是基于单颗纳米粒子的散射 的检测, 而溶液中测量的紫外可见消光谱包含了纳 米粒子吸收和散射的信息, 因此我们需要进一步获 得单颗纳米粒子的散射信息. 接下来, 采用暗场光 谱仪和扫描电镜共定位的方法来研究不同折射率 基底对单颗纳米粒子散射性质的调控: 将合成的三 种不同比例的 $\mathrm{Au} @ \mathrm{Ag}$ 纳米粒子分别置于含标记的 二氧化硅、三氧化二铝和二氧化钛(折射率分别为 1.52、1.77、2.61)基底上, 在暗场显微镜下寻找研 究的纳米粒子, 然后通过Renishaw inVia进行单颗纳 米粒子的散射光谱信息采集，同时通过定位标记记 录纳米粒子在基底上的位置, 暗场显微镜采集光学 图像; 随后的SEM表征通过定位标记和暗场显微镜 采集到的光学图像寻找研究的纳米粒子, 获得相应 的电镜形貌图, 从而实现暗场和SEM共定位. 图6为 $7.5 \mathrm{~nm} \mathrm{Au} @ 28 \mathrm{~nm} \mathrm{Ag}$ 纳米粒子的暗场图像及相对 应的电镜图, 三种不同比例的 $\mathrm{Au} @ \mathrm{Ag}$ 纳米粒子在不
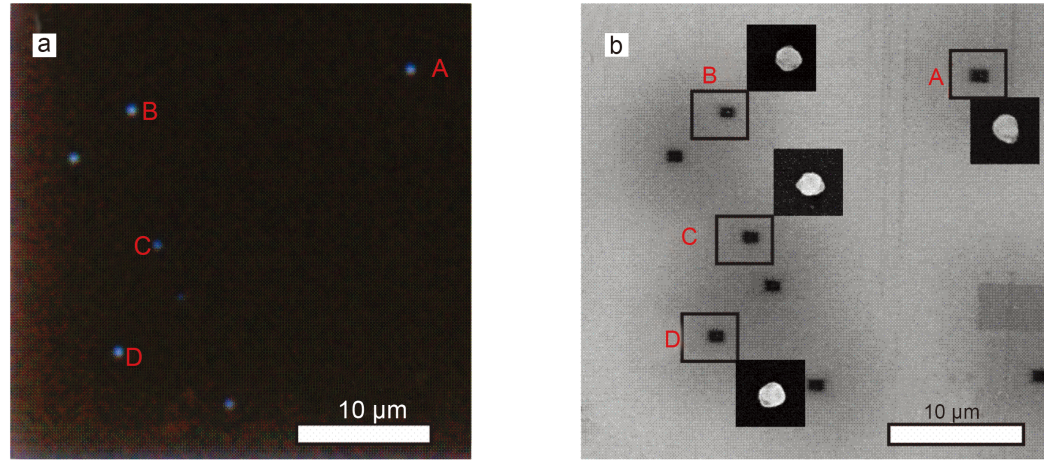

图6 $7.5 \mathrm{~nm} \mathrm{Au@28} \mathrm{nm} \mathrm{Ag}$ 的纳米粒子置于二氧化硅基底上的暗场散射光图像(a)及相对应的SEM图(b)

Fig.6 Dark-filed light scattering images and the corresponding SEM images of $7.5 \mathrm{~nm} \mathrm{Au@28} \mathrm{nm} \mathrm{Ag} \mathrm{NPs} \mathrm{on}$ the $\mathrm{SiO}_{2}$ substrate 

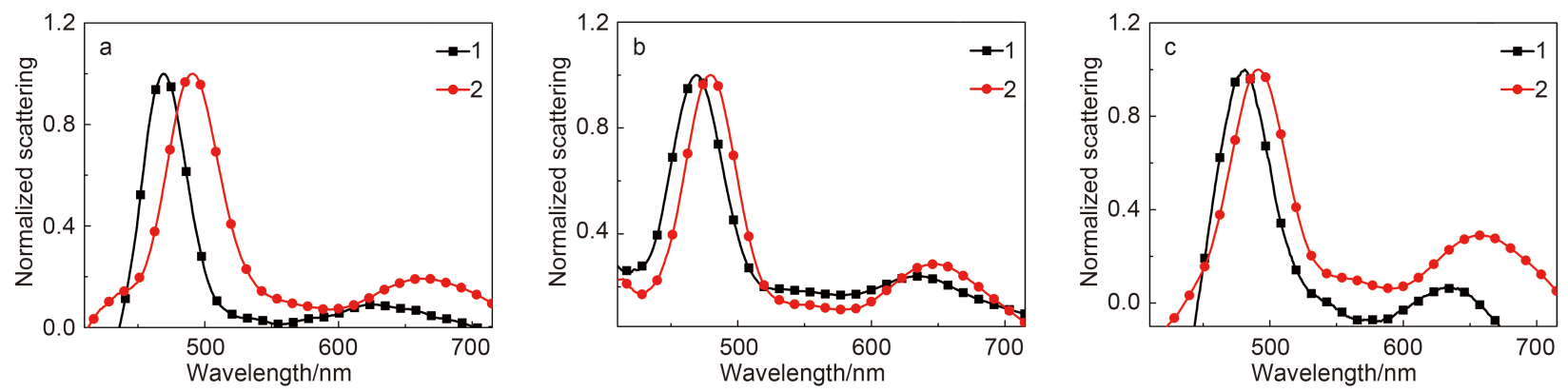

图7 不同核壳比的 $\mathrm{Au} @ \mathbf{A g}$ 纳米粒子在二氧化硅、三氧化二铝基底上的暗场散射光谱

Fig.7 Dark-field scattering spectra of $\mathrm{Au@Ag} \mathrm{NPs} \mathrm{with} \mathrm{different} \mathrm{core-shell} \mathrm{ratios} \mathrm{on} \mathrm{the} \mathrm{SiO}_{2}$ and $\mathrm{Al}_{2} \mathrm{O}_{3}$ substrates

(a) 7.5 nm Au@28nm Ag; (b) 16 nm Au@20.5 nm Ag; (c) 26 nm Au@10 nm Ag; (1) $\mathrm{SiO}_{2}$ substrate, (2) $\mathrm{Al}_{2} \mathrm{O}_{3}$ substrate

同基底的散射光谱都是通过此种方法采集, 以确定 所采集到LSPR信号为单颗纳米粒子的散射光谱, 同 时每种粒子的单颗粒信号至少采集 10 颗, 以确保数 据的可靠性. 但在二氧化钛基底采集到的单颗粒散 射光谱很弱, 原因未知有待后续研究.

图7为7.5 nm Au@28 nm Ag, 16 nm Au@20.5 $\mathrm{nm} \mathrm{Ag}, 26 \mathrm{~nm} \mathrm{Au} @ 10 \mathrm{~nm} \mathrm{Ag}$ 三种纳米粒子在二氧 化硅和三氧化二铝基底上的暗场散射光谱(信号强 度经归一化处理). 对于7.5 nm Au@28 nm Ag和16

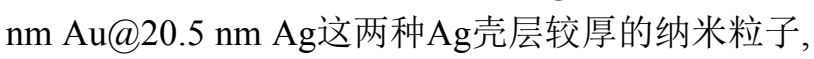
散射性质与 $\mathrm{Ag}$ 纳米球相似. 而对于 $26 \mathrm{~nm} \mathrm{Au@10}$ $\mathrm{nm} \mathrm{Ag}$ 纳米粒子, 虽然 $\mathrm{Ag}$ 壳层较薄, 但是 $52 \mathrm{~nm}$ 的 $\mathrm{Au}$ 核以吸收为主, ${ }^{11}$ 所以收集的光谱为 $\mathrm{Ag}$ 壳层的散 射光谱. 图中同时可看出单颗纳米粒子的半峰宽较 小 $(40 \mathrm{~nm}$ 左右), 且三种纳米粒子在 $650 \mathrm{~nm}$ 左右处出 现一个峰, 这主要是 $\mathrm{Au}$ 和 $\mathrm{Ag}$ 的耦合峰. 三种纳米粒 子在折射率不同的基底上产生了位移, 其中7.5 nm Au@ $28 \mathrm{~nm} \mathrm{Ag}$ 纳米粒子的位移程度最大. 这一纳米结构 符合对激发光无选择性的球形纳米粒子, 且其大小 能使制备的针尖兼顾高空间分辨和高性噪比, 同时 还有较高的介电敏感性. 综上考虑7.5 nm Au@28 $\mathrm{nm} \mathrm{Ag}$ 核壳纳米结构可作为下一步研究所采用的候 选纳米结构之一.

\section{4 结 论}

将 $\mathrm{CO}_{3}^{2-}$ 阴离子与 $\mathrm{Ag}^{+}$形成的前驱体引入到对 $\mathrm{Au}$ 核的 $\mathrm{Ag}$ 壳层包覆中, 一步法合成具有较厚的 $\mathrm{Ag}$ 壳层的、尺寸均一性好的 $\mathrm{Au} @ \mathrm{Ag}$ 纳米粒子. 通 过控制每次加入 $\mathrm{Ag}$ 的前驱体与 $\mathrm{Au}$ 种之比, 可较为精 确地实现不同厚度的Ag壳层包覆, 从而获得核壳比 不同的 $\mathrm{Au} @ \mathrm{Ag}$ 纳米粒子. 我们将合成的三种比例的 纳米粒子分别置于不同的折射率溶液中, 考察它们
对环境介质变化的敏感性. 我们还将三种核壳比的 $\mathrm{Au} @ \mathrm{Ag}$ 纳米粒子置于折射率不同的基底上, 研究其 单颗纳米粒子的散射光学性质. 最后证实了 $7.5 \mathrm{~nm}$ $\mathrm{Au} @ 28 \mathrm{~nm} \mathrm{Ag}$ 纳米粒子对于环境介质变化最为敏 感, 有望进一步应用于纳米间隙耦合增强表面等离 激元共振扫描显微探针上.

\section{References}

(1) Willets, K. A.; Van Duyne, R. P. Annu. Rev. Phys. Chem. 2007, 58, 267. doi: 10.1146/annurev.physchem. 58.032806.104607

(2) Mayer, K. M.; Hafner, J. H. Chem. Rev. 2011, 111, 3828. doi: $10.1021 / \mathrm{cr} 100313 \mathrm{v}$

(3) Anker, J. N.; Hall, W. P.; Lyandres, O.; Shah, N. C.; Zhao, J.; Van Duyne, R. P. Nat. Mater. 2008, 7, 442. doi: 10.1038/nmat2162

(4) Li, Y.; Jing, C.; Zhang, L.; Long, Y. T. Chem. Soc. Rev. 2012, 41, 632. doi: 10.1039/c1cs15143f

(5) Ciracì, C.; Hill, R. T.; Mock, J. J.; Urzhumov, Y.; FernándezDomínguez, A. I.; Maier, S. A.; Pendry, J. B.; Chilkoti, A.; Smith, D. R. Science 2012, 337, 1072. doi: 10.1126/science. 1224823

(6) Tian, Z. Q.; Wang, F. F.; Zhan, D. P.; Zhou, J. Z. Tip Enhanced Dark Field Microscopy, Electrochemical System and Leveling Device. CN Patent 102798735A, 2012-11-28. [田中群, 王芳芳, 詹东平, 周剑章. 针尖增强暗场显微镜、电化学测试装置和调 平系统: 中国, CN102798735A[P]. 2012-11-28.]

(7) Chen, H. J.; Kou, X. S.; Yang, Z.; Ni, W. H.; Wang, J. F. Langmuir 2008, 24 (10), 5233. doi: 10.1021/la800305j

(8) Awada, C.; Popescu, T.; Douillard, L.; Charra, F.; Perron, A.; Yockell-Lelièvre, H.; Baudrion, A. L.; Adam, P. M.; Bachelot, R. J. Phys. Chem. C 2012, 116 (27), 14591. doi: 10.1021/jp303475c

(9) Knight, M. W.; Wu, Y. P.; Lassiter, J. B.; Nordlander, P.; Halas, N. J. Nano Lett. 2009, 9 (5), 2188. doi: 10.1021/n1900945q

(10) Young, M. A.; Die ringer, J. A.; Van Duyne, R. P. Plasmonic Materials for Surface-Enhanced and Tip-Enhanced Raman Spectroscopy. In Tip Enhancement; Kawata, S., Shalaev, V. M. Eds.; 1st ed. Elsevier Science Ltd.: Amsterdam, the Netherlands, 
2007; pp 3-4.

(11) Ziegler, C.; Eychmüller, A. J. Phys. Chem. C 2011, 115 (11), 4502. doi: $10.1021 / \mathrm{jp} 1106982$

(12) Evanoff, D. D., Jr.; Chumanov, G. J. Phys. Chem. B 2004, 108 (37), 13948. doi: $10.1021 / \mathrm{jp} 047565 \mathrm{~s}$

(13) Liu, B. J.; Lin, K. Q.; Hu, S.; Wang, X.; Lei, Z. C.; Lin, H. X.; Ren, B. Anal. Chem. 2015, 87 (37), 1058. doi: 10.1021/ac503612b

(14) West, P. R.; Ishii, S.; Naik, G.; Emani, N.; Shalaev, V. M.; Boltasseva, A. Laser \& Photonics Reviews 2010, 4 (6), 1. doi: 10.1002/lpor.200900055

(15) Johnson, P. B.; Christy, R. W. Phys. Rev. B 1972, 6 (12), 4370.

(16) Jakab, A.; Rosman, C.; Khalavka, Y.; Becker, J.; Trügler, A.; Hohenester, U.; Sönnichsen, C. ACS Nano 2011, 5 (9), 6880. doi: $10.1021 / \mathrm{nn} 200877 \mathrm{~b}$

(17) Sun, Y. G. Chem. Soc. Rev. 2013, 42, 2497. doi: 10.1039/c2cs35289c

(18) Li, H. S.; Xia, H. B.; Ding, W. C.; Li, Y. J.; Shi, Q. R.; Wang, D. Y.; Tao, X. T. Langmuir 2014, 30 (9), 2498. doi: 10.1021/la4047148

(19) Sondi, I.; Goia, D. V.; Matijević, E. J. Colloid Interf. Sci. 2003, 260 (1), 75. doi: 10.1016/\$0021-9797(02)00205-9

(20) Panáček, A.; Kvítek, L.; Prucek, R.; Kolář, M.; Večeřová, R.; Pizúrová, N.; Sharma, V. K.; Nevěčná, T.; Zbořil, R. J. Phys.
Chem. B 2006, 110 (33), 16248. doi: 10.1021/jp063826h

(21) Frens, G. Nature 1973, 241, 20. doi: 10.1038/physci241020a0

(22) Sqalli, O.; Bernal, M. P.; Hoffmann, P.; Marquis-Weible, F. Appl. Phys. Lett. 2000, 76, 2134. doi: 10.1063/1.126277

(23) Zhang, X.; Wang, H.; Su, Z. H. Langmuir 2012, 28 (44), 15705. doi: $10.1021 / 1 \mathrm{a} 303320 \mathrm{z}$

(24) Liu, B. H.; Han, G. M.; Zhang, Z. P.; Liu, R. Y.; Jiang, C. L.; Wang, S. H.; Han, M. Y. Anal. Chem. 2011, 84 (1), 255. doi: 10.1021/ac202452t

(25) Lim, D. K.; Kim, I. J.; Nam, J. M. Chem. Commun. 2008, 42, 5312. doi: 10.1039/b810195g

(26) Rodríguez-González, B.; Burrows, A.; Watanabe, M.; Kiely, C. J.; Liz-Marzán, L. M. J. Mater. Chem. 2005, 15, 1755.

(27) Samal, A. K.; Polavarapu, L.; Rodal-Cedeira, S.; Liz-Marzán, L. M.; Pérez-Juste, J.; Pastoriza-Santos, I. Langmuir 2013, 29 (48), 15076. doi: 10.1021/la403707j

(28) Mock, J. J.; Smith, D. R.; Schultz, S. Nano Lett. 2003, 3 (4), 485. doi: $10.1021 / \mathrm{n} 10340475$

(29) Sun, Y. G.; Xia, Y. N. Anal. Chem. 2002, 74 (20), 5297. doi: 10.1021/ac0258352

(30) Sherry, L. J.; Chang, S. H.; Schatz, G. C.; Van Duyne, R. P.; Wiley, B. J.; Xia, Y. N. Nano Lett. 2005, 5 (10), 2034. doi: $10.1021 / \mathrm{nl} 0515753$ 